\title{
CARACTERIZACIÓN FISICOQUÍMICA Y EVALUACIÓN DE LA ACTIVIDAD ANTIOXIDANTE DE PROPÓLEOS RECOLECTADOS EN EL DEPARTAMENTO DEL ATLÁNTICO, COLOMBIA
}

\section{PHYSICOCHEMICAL CHARACTERIZATION AND EVALUATION OF ANTIOXIDANT ACTIVITY OF PROPOLIS COLLECTED IN THE ATLÁNTIC DEPARTMENT, COLOMBIA}

\author{
Yeray Rodríguez ${ }^{1}$, Fredys Sánchez-Catalán ${ }^{2}$, Benjamín Rojano ${ }^{3}$, Diego Durango ${ }^{4}$, Jesús Gil ${ }^{5}$, Juan Marín-Loaiza ${ }^{6}$
}

${ }^{1}$ Químico, Estudiante Maestría en Ciencias-Química. Facultad de Ciencias. Universidad Nacional de Colombia. Medellín, Antioquia, Colombia. yarodriguezn@unal.edu.co; ${ }^{2}$ Químico, Estudiante Maestría en Ciencias-Química. Facultad de Ciencias. Universidad Nacional de Colombia. Medellín, Antioquia, Colombia. fsanchezca@unal.edu.co; ${ }^{3}$ Químico. M.Sc. Ph.D. Facultad de Ciencias. Universidad Nacional de Colombia. Medellín, Antioquia, Colombia. brojano@unal.edu.co; ${ }^{4}$ Químico. M.Sc. Facultad de Ciencias. Universidad Nacional de Colombia. Medellín, Antioquia, Colombia. dldurango@unal.edu.co; ${ }^{5}$ Químico. Ph.D. Facultad de Ciencias Agropecuarias. Universidad Nacional de Colombia. Medellín, Antioquia, Colombia. jhgilg@unal. edu.co; ${ }^{6}$ Químico Farmacéutico. M.Sc. Ph.D. Departamento de Farmacia. Facultad de Ciencias. Universidad Nacional de Colombia. Bogotá, Colombia. Carrera 30 No.45-03. Edificio 450. jcmarinlo@unal.edu.co

Rev. U.D.C.A Act. \& Div. Cient. 15(2): 303 - 311, 2012

\section{RESUMEN}

Se realizó una comparación de las propiedades fisicoquímicas de los propóleos recolectados en los municipios de Galapa, Sabanalarga, Santo Tomás y Juan de Acosta, del departamento del Atlántico (Colombia). Adicionalmente, se estableció la actividad antioxidante in vitro de los extractos etanólicos de propóleos (EEP), mediante las técnicas de DPPH, ABTS, FRAP y ORAC. El análisis fisicoquímico de los propóleos mostró valores de 8,48 - 24,21\%, 1,77 - 6,07\%, 55,57 - 77,93\%, 5,74 - 11,69\% y 0,35 - 3,86\%, para el EEP, ceras, insolubles, humedad y cenizas, respectivamente. La determinación para los fenoles totales, se encuentra entre 63,72 y 94,55mg, de ácido gálico/g de muestra. Las absorbancias específicas de los EEP estuvieron dentro del rango de 26 a 96 y el contenido de flavonoides totales mostró valores entre 1,90 y 3,36mg, de quercetina/g de muestra. De los extractos, el que presentó mayor capacidad antioxidante fue el propóleos, recolectado en el municipio de Galapa, con los siguientes valores DPPH: 190,41 TEAC $(\mu \mathrm{mol} / \mathrm{g})$, ABTS: $1918,41 \mathrm{TEAC}(\mu \mathrm{mol} / \mathrm{g})$ y FRAP: 321,27 AEAC $(\mu \mathrm{mol} / \mathrm{g})$ y el segundo valor más alto en ORAC (1964,80 $\mu \mathrm{mol}$ Trolox/g de propóleos). Los resultados obtenidos indican que los propóleos estudiados presentaron variación en las propiedades fisicoquímicas. Los valores de algunos de los parámetros fi- sicoquímicos estimados no cumplieron con las normativas de calidad establecidas internacionalmente; no obstante, los extractos etanólicos de propóleos evaluados presentaron un alto contenido de fenoles, los cuales, están relacionados con las propiedades antioxidantes encontradas experimentalmente.

Palabras clave: Propóleos, compuestos fenólicos, caracterización fisicoquímica, capacidad antioxidante.

\section{SUMMARY}

A comparison of the physicochemical properties of the propolis collected in the Galapa, Sabanalarga, Santo Tomás and Juan de Acosta's municipalities of the Atlantic department (Colombia) was made. Additionally, the in vitro's antioxidant activity of ethanol extracts of propolis (EEP) through the DPPH, ABTS, FRAP and ORAC methods was established. The physicochemical analysis of propolis indicated values between $8.48-24.21 \%, 1.77-6.07 \%$, 55.57-77.93\%, 5.74$11.69 \%$ and $0.35-3.86 \%$, for EEP, waxes, insoluble residues, humidity and ashes respectively. The determination for the total phenolic content was between 63.72 and $94.55 \mathrm{mg}$ of gallic acid/g of sample. The EEP's specific absorbance 
ranged from 26 to 96 and the total flavonoid content showed values between 1.90 and $3.36 \mathrm{mg}$ of quercetin $/ \mathrm{g}$ of sample. The extract that showed the highest antioxidant capacity was the propolis collected in the municipality of Galapa with the following values, DPPH: 190,41 TEAC $(\mu \mathrm{mol} / \mathrm{g})$, ABTS: 1918,41 TEAC $(\mu \mathrm{mol} / \mathrm{g})$, FRAP: 321,27 AEAC $(\mu \mathrm{mol} / \mathrm{g})$, and the second highest value in ORAC $(1964,80 \mu \mathrm{mol} \mathrm{TROLOX/g}$ of propolis). The results obtained indicated that the propolis studied showed a variation in its physicochemical properties. Some of the estimated physicochemical parameter values did not fulfill the established international quality regulations. Nevertheless, the ethanol extracts of the evaluated propolis presented a high content of phenols, related with the antioxidant properties found experimentally.

Key words: Propolis, phenolic compounds, physicochemical properties, antioxidant properties.

\section{INTRODUCCIÓN}

Las abejas recolectan exudados de varias especies de plantas, que al mezclarlos con polen y sus secreciones enzimáticas generan un material resinoso, conocido como propóleos (Kalogeropoulos et al. 2009). La función primordial de este producto natural es actuar como un agente sellante, para mantener la temperatura de la colmena y protegerla de agentes invasores y microorganismos (Sforcin \& Bankova, 2011). Adicional a estas propiedades, para el propóleos se han demostrado actividades biológicas y farmacológicas, tales como: anti-inflamatoria (McLennan et al. 2008), antioxidante (Yang et al. 2011), antiproliferativa (Russo et al. 2004), hepatoprotectora (Banskota et al. 2001) e inmunomoduladora (Sforcin, 2007).

El propóleos contiene, aproximadamente, entre un 50-55\%, de resina y bálsamos; $30 \%$, de cera; $10 \%$, de volátiles y $5 \%$, de polen (Libério et al. 2009). El 5\% restante corresponde a una gran variedad de compuestos que van desde metabolitos secundarios, como polifenoles, quinonas sesquiterpénicas, cumarinas, terpenoides, hasta esteroides, aminoácidos, ácidos grasos y compuestos inorgánicos (Bankova et al. 2000). Adicionalmente, un gran número de estudios confirman que la composición química de los propóleos es altamente compleja y varía de acuerdo con la vegetación circundante, el periodo y la zona donde fueron recolectados, así como la especie de abeja que lo produce (Bermúdez-Camps et al. 2000; Kujumgiev et al. 1999; Marcucci, 1995).

Existe un elevado número de artículos en que se describe la caracterización fisicoquímica y la evaluación de las diversas actividades biológicas de propóleos obtenidos en países de los continentes Europeo (Valente et al. 2011), Asiático (Ahn et al. 2007), Africano (Petrova et al. 2010) y America- no (Russo et al. 2004), llegando a la conclusión que existen diferencias evidentes en cuanto a composición cualitativa y cuantitativa de actividad, aun cuando las muestras analizadas sean recolectadas en el mismo país (Koo et al. 1999). En la literatura disponible son escasas y dispersas las publicaciones relacionadas con el estudio de propóleos colombianos (Ferreira-Bastos et al. 2011; Palomino-García et al. 2009; Salamanca-Grosso et al. 2007; Ortega et al. 2011); debido a esto y con miras a establecer una norma de calidad que permita estandarizar el propóleos como insumo para la elaboración de diversos productos, consideramos importante realizar estudios sobre las características de los propóleos, que en Colombia han sido poco estudiados, como es el caso de muestras de propóleos provenientes del departamento del Atlántico. Estas caracterizaciones podrían conllevar al conocimiento de nuevos constituyentes con actividades biológicas promisorias (Popova et al. 2011).

En el presente trabajo, se reporta la caracterización fisicoquímica (cenizas, ceras, humedad, material insoluble, EEP, fenoles, flavonoides totales) y los análisis de la actividad antioxidante (DPPH, ABTS, FRAP y ORAC), de propóleos recolectados en los municipios de Galapa, de Juan de Acosta, de Sabanalarga y de Santo Tomás, pertenecientes al departamento del Atlántico-Colombia.

\section{MATERIALES Y MÉTODOS}

Recolección del propóleos. Los propóleos de abejas Apis mellifera africanizada, se obtuvieron de apiarios, del departamento del Atlántico, provenientes de los municipios de Sabanalarga (Latitud: $10^{\circ} 38^{\prime} \mathrm{N}$, Longitud: $74^{\circ} 55^{\prime} \mathrm{O}$; 108 msnm), de Galapa (Latitud: $10^{\circ} 53^{\prime} \mathrm{N}$, Longitud: 74 $53^{\prime} \mathrm{O}$; 50 msnm), de Juan de Acosta (Latitud: $10^{\circ} 50^{\prime} \mathrm{N}$, Longitud: $72^{\circ} 03^{\prime} \mathrm{O}$; $50 \mathrm{msnm}$ ) y de Santo Tomás (Latitud: $10^{\circ} 46^{\prime} \mathrm{N}$, Longitud: $74^{\circ} 55^{\prime} \mathrm{O}$; $8 \mathrm{msnm}$ ). Las muestras, se colectaron en el segundo trimestre de 2009 (periodo de sequía), por el método de raspado, empleando espátulas para remover el producto adherido en las caras laterales (tapa y entretapa), de cada colmena. El muestreo, se efectuó en forma aleatoria en diferentes colmenas de cada apiario, hasta formar una muestra compuesta representativa y en cantidad suficiente, para realizar los análisis. Los propóleos crudos, se maceraron y se les retiraron las impurezas mecánicas.

Obtención de los extractos: $30 \mathrm{~g}$ de muestra, se sometieron a extracción sucesiva con etanol del $96 \% \mathrm{v} / \mathrm{v}(3 \times 100 \mathrm{~mL})$, durante 48 horas, a temperatura ambiente y en ausencia de luz; luego, el material se cribó y el filtrado fue enfriado a $-18^{\circ} \mathrm{C}$, para precipitar las ceras.; el extracto, se llevó a sequedad en un evaporador rotatorio a presión reducida y una temperatura de $40^{\circ} \mathrm{C}$; los extractos etanólicos de propóleos (EEP), se almacenaron en viales ámbar y se preservaron a $-18^{\circ} \mathrm{C}$. 
Caracterización fisicoquímica: Para la caracterización fisicoquímica del propóleos crudo, se siguieron los métodos recomendados en la Norma del Ministerio de Agricultura de Brasil (Ministerio de Agricultura de Brasil-APACAME, 1999).

Absorbancia específica del espectro UV: La absorbancia específica del espectro UV de cada muestra de EEP, se obtuvo siguiendo el método propuesto por Miyataka et al. (1997).

Contenido de fenoles y flavonoides totales: El contenido de fenoles totales, se determinó por el método colorimétrico de Singleton \& Rossi (1965), con algunas modificaciones (Palomino-García et al. 2009); para construir la curva de calibración, se usaron soluciones de ácido gálico (SigmaAldrich) entre 50 y $500 \mu \mathrm{g} / \mathrm{mL}$ y los resultados se expresaron como mg, equivalentes de ácido gálico (GAE)/g de EEP.

El contenido de flavonoides totales en el EEP fue determinado por el método de Kumazawa et al. (2004); para construir la curva de calibración, se usaron soluciones de quercetina (Sigma-Aldrich), entre 5 y $25 \mu \mathrm{g} / \mathrm{mL}$ y el contenido total fue calculado como $\mathrm{mg}$, equivalentes de quercetina $(\mathrm{QE}) / \mathrm{g}$ de EEP.

Capacidad antioxidante: La actividad antioxidante de los extractos, se evaluó mediante la capacidad captadora del radical 1,1-difenil-2-picril hidracilo (DPPH') y se utilizó la metodología de Rojano et al. (2008). Los resultados fueron convertidos a porcentaje de inhibición y expresados como capacidad antioxidante en $\mu \mathrm{mol}$, de equivalentes de Trolox (ácido 6-hidroxi-2,5,7,8-tetrametilcromo-2-carboxílico; TE)/g de EEP (TEAC). El antioxidante sintético butil-hidroxitolueno (BHT), se usó como sustancia de referencia externa.

Para establecer la actividad inhibidora del radical 2,2'-azinobis (3-etilbenzotiazolina)- 6 sulfonato de amonio (ABTS ${ }^{\cdot+}$ ), se empleó el método propuesto por Aubad et al. (2007). Se utilizó una curva de calibración de Trolox como estándar y los resultados, se expresaron como capacidad antioxidante en $\mu$ mol de equivalentes Trolox (TE)/g de EEP (TEAC). Se usó como sustancia de referencia externa el BHT.

La actividad antioxidante reductora de $\mathrm{Fe}^{3+}$ (FRAP), se llevó a cabo según el método de Benzie \& Strain (1996); se utilizó una curva de calibración de ácido ascórbico (AA) y las actividades de los extractos de propóleos se expresaron como $\mu \mathrm{mol}$ de equivalentes de ácido ascórbico (AAE)/g de EEP (AEAC). Los valores, se expresaron como la media de tres réplicas; como sustancia de referencia, se empleó el antioxidante BHT.

El procedimiento experimental del ensayo ORAC estuvo basado en la metodología reportada por Ou et al. (2001), con algunas modificaciones (Naranjo et al. 2011). Se empleó
Trolox, como sustancia de referencia.

Los datos mostrados en las tablas son el resultado promedio de tres réplicas y son presentados como la media \pm su error estándar. Los datos fueron sometidos a un análisis de varianza (ANOVA), para establecer si había diferencias significativas entre las medias. Los resultados son expresados como valores de probabilidad con una $\mathrm{p}<0.05$, adoptada como criterio de significancia. Para la evaluación antioxidante, se llevó a cabo un análisis de varianza de una sola vía con tres repeticiones y una prueba de comparaciones múltiples de medias de $\mathrm{n}$ tratamientos, con un control de Dunnett, para comparar las muestras con la referencia; el valor de $p<0.05$ fue considerado significativo. Todos los cálculos se realizaron en el programa estadístico SPSS Statistics 17.0.

\section{RESULTADOS Y DISCUSIÓN}

Caracterización fisicoquímica: Los resultados obtenidos en la caracterización fisicoquímica de los propóleos del departamento del Atlántico, se ilustran en la tabla 1 . El valor del EEP es un parámetro que se relaciona directamente con la calidad del propóleos crudo, debido a que con este extracto se obtienen resinas solubles en alcohol, que es donde se presume están contenidas las sustancias biológicamente activas, que le brindan las características particulares a la muestra.

La norma brasilera señala que el porcentaje de EEP debe ser mínimo 35\%. Para las muestras analizadas, los porcentajes de EEP están por debajo (8,48 hasta $24,21 \%$ p/p), de lo que indica la norma; el valor más alto correspondió al propóleos de Galapa. Un resultado similar (14,08\%) fue obtenido por Palomino-García et al. (2010), cuando analizaron el propóleos del municipio de La Unión (Antioquia). Algunos autores atribuyen las diferencias en el contenido de resinas a diversos factores, tales como: la vegetación circundante, el periodo y la zona de cosecha, así como la especie de abeja que lo produce (Bermúdez-Camps et al. 2000; Kujumgiev et al. 1999; Marcucci, 1995).

Otro parámetro que se emplea como indicador de calidad es el porcentaje de humedad, ya que valores altos reflejan condiciones no apropiadas de manipulación y de almacenamiento (Cunha et al. 2004); además, se pueden generar condiciones propicias para el crecimiento microbiano (Carpes et al. 2009). Los valores de porcentaje de humedad de los propóleos analizados fueron de 5,74\% (Sabanalarga), 8,68\% (Santo Tomás), 10,51\% (Juan de Acosta) y 11,69\% (Galapa). La norma brasilera reporta que el porcentaje de humedad no debe ser mayor del $8 \%$; de acuerdo con esto, únicamente el propóleos recolectado en el municipio de Sabanalarga está dentro de los valores permitidos. 
Tabla 1. Evaluación de las propiedades fisicoquímicas de las muestras de propóleos recolectadas en el Departamento del Atlántico.

\begin{tabular}{|c|c|c|c|c|c|}
\hline \multirow{2}{*}{$\begin{array}{l}\text { Característica } \\
\text { fisicoquímica }\end{array}$} & \multicolumn{5}{|c|}{ Propóleos } \\
\hline & Galapa & Sabana larga & Santo Tomás & Juan de Acosta & $\begin{array}{c}\text { Norma Brasilera } \\
(\%)^{*}\end{array}$ \\
\hline $\operatorname{EEP}(\%)$ & $24,2 \pm 1,7^{a}$ & $8,5 \pm 2,7^{b}$ & $12,6 \pm 0,3^{c}$ & $15,6 \pm 0,9^{c}$ & Mínimo 35,0 \\
\hline Ceras (\%) & $5,2 \pm 1,7^{\mathrm{a}}$ & $1,8 \pm 0,0^{b}$ & $4,6 \pm 0,4^{a}$ & $6,1 \pm 0,5^{\mathrm{a}}$ & Máximo 25,0 \\
\hline Impurezas mecánicas (\%) & $55,6 \pm 3,7^{a}$ & $77,9 \pm 2,9^{b}$ & $65,3 \pm 3,3^{c}$ & $71,2 \pm 1,3^{\mathrm{d}}$ & Máximo 40,0 \\
\hline Humedad (\%) & $11,7 \pm 0,7^{\mathrm{a}}$ & $5,7 \pm 0,6^{b}$ & $8,7 \pm 0,3^{c}$ & $10,5 \pm 1,1^{a}$ & Máximo 8,0 \\
\hline Cenizas (\%) & $3,9 \pm 1,0^{\mathrm{a}}$ & $1,8 \pm 0,3^{b}$ & $0,4 \pm 0,3^{c}$ & $2,3 \pm 0,6^{b}$ & Máximo 5,0 \\
\hline
\end{tabular}

\# Todas las determinaciones están dadas en g/100 g de propóleos

*Requisitos de calidad exigidos de acuerdo con la Normatividad Brasilera (Ministerio de Agricultura de Brasil-APACAME, 1999).

Cada valor corresponde al promedio de tres experimentos diferentes \pm error estándar. En una misma fila, los valores seguidos por la misma letra no presentan diferencias significativas. El nivel de significancia es $\mathrm{P}<95 \%$.

En el propóleos no es recomendable un alto porcentaje de material insoluble y ceras, ya que la presencia de estos va en detrimento de la calidad al no hacer ningún aporte significativo a la actividad biológica. Para el material insoluble (impurezas mecánicas), los propóleos del departamento del Atlántico presentaron valores muy superiores (55-78\%) a los permitidos por la norma brasilera (40\%) (Tabla 1), lo que puede estar relacionado con la metodología de colecta empleada por el apicultor (Sawaya et al. 2011).

Contrario a lo obtenido para los otros parámetros determinados, el análisis de porcentaje de ceras mostró que todas las muestras están dentro del rango de valores, propuesto por la normatividad brasilera (máximo 25\%). El propóleos con menor porcentaje de ceras fue el recolectado en Sabanalarga. Asimismo, el porcentaje de cenizas de los cuatro propóleos analizados se encuentra por debajo del valor máximo permitido (5\%), que cumple con lo establecido en la norma. En este caso, la muestra con menor porcentaje de cenizas fue el propóleos procedente de Santo Tomás.

Contenido de fenoles y flavonoides totales: En el propóleos, la presencia de fenoles y flavonoides se asocia con la actividad biológica y se considera como un indicador de calidad por los entes reguladores de la producción de propóleos en países, como Brasil (Ministerio de Agricultura de BrasilAPACAME, 1999) y Argentina (Norma IRAM-INTA, 2004). Los resultados consignados en la tabla 2 indican que el contenido de compuestos fenólicos osciló entre valores que van desde 63,72 hasta $94,55 \mathrm{mg}$ de GAE/g de EEP. La muestra de Sabanalarga presentó el menor contenido de compuestos fenólicos.
Los propóleos de Juan de Acosta, de Santo Tomás y de Galapa presentaron un contenido fenólico mayor, al reportado para propóleos del departamento de Antioquia-Colombia, que estuvieron en un rango comprendido entre 22,11 a 75,22 mg GAE/g de EEP (Palomino-García et al. 2009). Finalmente, todas las muestras analizadas superan el requisito mínimo (50mg de GAE/g de EEP), establecido por la legislación brasilera para la presencia de compuestos fenólicos, como determinante de la calidad.

Adicionalmente, dentro del grupo de los polifenoles, se encuentran los flavonoides, los cuales, son reconocidos por sus propiedades antioxidantes y se les atribuye, en gran parte, aunque no exclusivamente, la actividad biológica del propóleos (Libério et al. 2009). Los valores obtenidos para el contenido de flavonoides en los extractos estuvieron entre 1,90 y $5,16 \mathrm{mg}$ de $\mathrm{QE} / \mathrm{g}$ de EEP. De todas las muestras evaluadas, la única que cumple con la norma brasilera $(5 \mathrm{mg} / \mathrm{g})$ es el propóleos de Santo Tomás (5,16mg/g de EEP, Tabla 2$)$, la cual, a su vez presentó el segundo mayor contenido de fenoles $(86,63 \mathrm{mg} / \mathrm{g}$ de EEP).

El propóleos de Juan de Acosta, a pesar de presentar el mayor contenido de fenoles totales (94,55mg de GAE/g de EEP), tiene el menor valor de contenido de flavonoides $(1,90 \mathrm{mg}$ de $\mathrm{QE} / \mathrm{g}$ de EEP), lo cual, indica una mayor distribución de fenoles diferentes a los flavonoides. Comparando los resultados de este análisis con los datos obtenidos por PalominoGarcia et al. 2009 (fenoles: 22,11-75,22mg de GAE/g EEP; flavonoides: 4,75-42,37mg QE/g EEP) y Salamanca-Grosso et al. 2007 (flavonoides totales: 6,30-22,33mg/g EEP), para otros propóleos colombianos, se puede considerar al propóleos de Santo Tomás como una muestra con un contenido 
apreciable de fenoles $(86,63 \pm 4,38 \mathrm{mg}$ de GAE/g EEP) y flavonoides $(5,16 \pm 0,21 \mathrm{mg}$ QE/g EEP); no obstante, el contenido de fenoles y flavonoides de los propóleos evaluados fue inferior a los valores reportados en muestras de otras regiones del mundo (Ahn et al. 2004; 2007; Kumazawa et al. 2004).

La variación en la composición de los propóleos ha sido descrita previamente y se puede deber, principalmente, a factores como las características geográficas y climáticas donde fueron colectadas las muestras, la vegetación a la que las abejas tienen acceso para elaborar la resina y la especie de abeja, entre otros (Bankova, 2005a). Estudios previos relacionados con la composición del propóleos en diferentes países han permitido concluir que aunque los flavonoides son las principales sustancias activas, también se pueden encontrar otro tipo de metabolitos (lignanos, benzofenonas preniladas, ácidos diterpénicos, ácidos coumáricos y cinámicos con sus respectivos ésteres), como los responsables de la actividad biológica (Bankova, 2005b). Para establecer una correlación adecuada entre la variación del contenido de fenoles y flavonoides y la procedencia de la muestra, se hace necesario realizar estudios más detallados con los propóleos del departamento del Atlántico, que conduzcan a establecer: la composición química, las plantas empleadas por las abejas para su producción y las propiedades biológicas de los mismos.

El valor de absorbancia específica ( $E_{1 \mathrm{~cm}}^{1 \%}$ ), calculada en el máximo de absorbancia del espectro de absorción UV del EEP, es un parámetro usado para evaluar la calidad del propóleos (Kumazawa et al. 2004), debido a que varias de las actividades biológicas de este producto apícola se atribuyen a la presencia de compuestos fenólicos, flavonoides y ácidos caféicos, los cuales, presentan absorciones máximas entre las longitudes de onda de 240 y 350nm. Como se muestra en la tabla 2, los valores de absorbancia específica estuvieron en el rango de 26,54 (283nm) a 79,32 (291nm); siendo más bajos para las muestras de Juan de Acosta y de Sabanalarga, cuyo contenido de flavonoides también fue el menor. Resultados semejantes fueron previamente reportados por Kumazawa et al. (2004) para propóleos de Tailandia $\left(E_{1 \mathrm{~cm}}^{1 \%}\right.$ $=5$, determinado a $275 \mathrm{~nm})$ y África $\left(E_{1 \mathrm{~cm}}^{1 \%}=102\right.$, determinado $290 \mathrm{~nm}$ ). Además, todas las muestras presentaron una absorción máxima a longitudes de onda entre 274 y 291nm. Es posible inferir que las diferencias en la longitud de onda máxima correspondan a diversas composiciones de fenoles y flavonoides presentes en los propóleos, como consecuencia de los diferentes orígenes botánicos y geográficos.

Capacidad antioxidante: Al determinar la actividad antioxidante de los cuatro propóleos recolectados en el departamento del Atlántico, empleando los métodos de DPPH, ABTS, FRAP y ORAC, con el fin de establecer la presencia de algunos componentes con características antioxidantes en la muestra, se obtuvieron los resultados consignados en la tabla 3.

Los valores de inhibición del radical DPPH obtenidos muestran que el propóleos de Galapa fue el más activo $(190,41 \mu \mathrm{mol}$ TE/g de EEP), seguido por el de Santo Tomás $(134,18 \mu \mathrm{mol}$ TE/g de EEP) y el de Juan de Acosta $(103,79 \mu \mathrm{mol}$ TE/g de EEP). De las muestras analizadas, el propóleos de Sabanalarga fue el de más baja capacidad captadora del radical DPPH $(65,14 \mu \mathrm{mol}$ TE/g de EEP). Comparando estos valores con propóleos de diferentes regiones del departamento de Antioquia, se encontró que las muestras del Atlántico presentaron una actividad mayor que los propóleos de Caldas (CR: 54,6 TE/g de EEP) y el Apiario Lima (33,9 TE/g de EEP), pero fue inferior a la de los propóleos de Caldas (CT:324,6

Tabla 2. Contenido de fenoles totales y flavonoides en las muestras de propóleos.

\begin{tabular}{|c|c|c|c|}
\hline MUESTRA & $\begin{array}{c}\text { Coeficiente de extinción en el máximo de } \\
\text { absorbancia } E_{1 c m}^{1 \%}(\lambda)\end{array}$ & $\begin{array}{l}\text { Contenido de } \\
\text { Fenoles Totales }\end{array}$ & $\begin{array}{l}\text { Contenido de } \\
\text { Flavonoides Totales }\end{array}$ \\
\hline Galapa & 73,5 (290) & $81,2 \pm 10,0^{\mathrm{a}}$ & $3,4 \pm 0,8^{\mathrm{a}}$ \\
\hline Sabanalarga & $29,0(274,5)$ & $63,7 \pm 16,1^{b}$ & $2,3 \pm 0,1^{a}$ \\
\hline Santo Tomás & $79,3(291,5)$ & $86,6 \pm 4,4^{c}$ & $5,2 \pm 0,2^{a}$ \\
\hline Juan de Acosta & $26,5(283,5)$ & $94,6 \pm 21,1^{b}$ & $1,9 \pm 0,1^{a}$ \\
\hline
\end{tabular}

${ }^{\mathrm{A}} \mathrm{mg}$ de ácido gálico / g de muestra (mg de GAE/g de EEP).

${ }^{\mathrm{B}} \mathrm{mg}$ de quercetina / $\mathrm{g}$ de muestra ( $\mathrm{mg}$ de QE/g de EEP). Los valores representan la media de tres repeticiones \pm la desviación estándar.

En una misma columna, los valores seguidos por la misma letra no presentan diferencias significativas. El nivel de significancia es $\mathrm{P}<95 \%$. 
TE/g de EEP), de Betania (BR: 263,3 TE/g de EEP) y el estándar BHT (517,4 TE/g de EEP) (Palomino-García et al. 2009). Posiblemente, estas diferencias en la actividad se deban a las variaciones en composición de fenoles y flavonoides presentadas entre las muestras. En general, los propóleos del Atlántico presentaron una mayor concentración de fenoles y un contenido de flavonoides inferior, en comparación con los propóleos antioqueños.

Las muestras analizadas, mediante la técnica ABTS, presentaron un comportamiento similar al reportado para la prueba de DPPH, donde el propóleos de Galapa mostró la mayor decoloración del radical ABTS $(1918,41 \pm 44,45 \mu \mathrm{mol}$ trolox/g de EEP) y el propóleos de Sabanalarga la menor $(739,74 \pm 14,51 \mu \mathrm{mol}$ trolox $/ \mathrm{g}$ de EEP). Adicionalmente, se puede apreciar la relación que se da entre el contenido de compuestos fenólicos y la capacidad antioxidante de los extractos.

La actividad antioxidante esperada en mezclas puede ser el resultado de los posibles efectos sinérgicos, cooperativos o antagonistas de las sustancias presentes. Al tener en cuenta los resultados obtenidos, se puede inferir que no solo los fenoles son los responsables de la capacidad antioxidante, sino que puede haber otras sustancias implicadas (Huang et al. 2005).

Los valores de FRAP para las muestras de propóleos analizadas estuvieron entre 112,05 y 321,27 AEAC ( $\mu$ mol de ácido ascórbico/g de EEP) (Tabla 3). El propóleos de Galapa y el de Sabanalarga fueron las muestras con el mayor y el menor valor antioxidante en el ensayo de FRAP, conservándose la misma tendencia mostrada en las pruebas de actividad an- tioxidante (DPPH y ABTS). También se aprecia que ninguno de los extractos probados supera la capacidad antioxidante, mostrada por el estándar empleado (BHT).

En la tabla 3, se reportan los valores ORAC para los extractos etanólicos de propóleos, expresados como $\mu \mathrm{mol}$ de equivalente de Trolox/g de EEP. Los valores más altos de ORAC fueron 2211,94 y 1964,80umoles de Trolox/g de EEP, que corresponden a las muestras de Santo Tomás y de Galapa, respectivamente. El valor más bajo de ORAC fue para la muestra de Sabanalarga $(475,02 \mu$ moles de Trolox/g de EEP). Estos resultados son semejantes a los encontrados en análisis realizados para propóleos uruguayos (Silva et al. 2011), específicamente, para las muestras que fueron clasificadas en el grupo de bajo valor ORAC (1,8 - 2,6umol TE/ mg de EEP).

Los extractos de Galapa, de Santo Tomás y de Juan de Acosta fueron los que mostraron el mayor contenido de fenoles totales. De igual manera, el contenido de flavonoides fue mayor para el propóleos de Santo Tomás, seguido del de Galapa. Por otro lado, el extracto del municipio de Sabanalarga fue el que mostró menor contenido de compuestos fenólicos y el tercer valor en contenido de flavonoides (Tabla 2). En diversos estudios con propóleos provenientes de Europa y de Sur América se han correlacionado la capacidad antioxidantes, por diversas metodologías con el contenido de polifenoles y se ha reportado que estas sustancias no son las únicas responsables de la actividad (Ahn et al. 2007; Kumazawa et al. 2004; Sawaya et al. 2011). En este trabajo, se encontró que el contenido de fenoles no correlaciona significativamente (datos no presentados) con ninguna de las formas de medir la actividad antioxidante; sin embargo, el contenido de

Tabla 3. Actividad antioxidante de los extractos etanólicos de los propóleos recolectados en diferentes municipios del departamento del Atlántico, Colombia.

\begin{tabular}{|c|c|c|c|c|}
\hline Muestra & $\begin{array}{c}\text { DPPH } \\
\text { TEAC }^{\#} \pm \text { DE } \\
(\mu \mathrm{mol} / \mathrm{g} \text { EEP })\end{array}$ & $\begin{array}{c}\text { ABTS } \\
\text { TEAC }^{\#} \pm \text { DE } \\
(\mu \mathrm{mol} / \mathrm{g} \text { EEP })\end{array}$ & $\begin{array}{c}\text { FRAP } \\
\text { AEAC }^{\&} \pm \text { DE } \\
(\mu \mathrm{mol} / \mathrm{g} \text { EEP })\end{array}$ & $\begin{array}{c}\text { ORAC } \\
(\mu \mathrm{mol} \text { Trolox } / \mathrm{g} \\
\text { de EEP })\end{array}$ \\
\hline Galapa & $190,4 \pm 6,9^{a}$ & $1918,4 \pm 44,5^{a}$ & $321,3 \pm 55,6^{a}$ & 1964,8 \\
\hline Sabanalarga & $65,1 \pm 2,1^{b}$ & $739,7 \pm 14,5^{b}$ & $112,1 \pm 11,5^{b}$ & 475,0 \\
\hline Santo Tomás & $134,2 \pm 3,5^{c}$ & $1322,6 \pm 4,7^{c}$ & $262,7 \pm 24,5^{c}$ & 2211,9 \\
\hline Juan de Acosta & $103,8 \pm 2,5^{\mathrm{d}}$ & $1061,1 \pm 19,6^{d}$ & $191,1 \pm 31,5^{d}$ & 709,5 \\
\hline BHT (Estándar) & $517,4 \pm 0,1^{\mathrm{e}}$ & $884,9 \pm 0,1^{\mathrm{e}}$ & $500,6 \pm 5,5^{\mathrm{e}}$ & \\
\hline
\end{tabular}

\# Capacidad antioxidante en equivalentes Trolox (TE).

\& Capacidad antioxidante en equivalentes de ácido ascórbico. Los valores representan la media de tres repeticiones \pm la desviación estándar (DE).

En una misma columna, los valores seguidos por la misma letra no presentan diferencias significativas. El nivel de significancia es $\mathrm{P}<95 \%$. 
flavonoides correlaciona significativamente $(\mathrm{R} 2=83,4 \%$; $\mathrm{F}$ $=10)$ con los valores ORAC mediante la expresión:

$$
\text { Valor ORAC }=-8983,2+1814,7 \text { Ln (Fenoles Totales) }
$$

En conclusión, el fenómeno oxidativo exhibido por los propóleos del Atlántico puede ser debido a que los flavonoides atrapan radicales libres mediante un mecanismo de transferencia de protones, medidos por la metodología ORAC.

Los resultados entre los diferentes métodos de ensayo no son completamente comparables, dado que el fundamento de cada ensayo antioxidante varía. En particular, la muestra de Sabanalarga presentó los valores más bajos de actividad en los cuatro modelos evaluados, mientras que el propóleos de Galapa presentó la mayor capacidad antioxidante, exceptuando el resultado obtenido en ORAC.

Las muestras analizadas de propóleos crudo del departamento del Atlántico presentaron variaciones en las características fisicoquímicas; donde se observó que los contenidos de ceras y de cenizas se encontraron entre los valores establecidos por las normas internacionales. Esto permite realizar dos consideraciones: la primera, relacionada con las condiciones de recolección y de almacenamiento de los propóleos, que quizás no sean las más adecuadas y repercuten en el detrimento de la calidad de las muestras; y, la segunda, la diferencia de los resultados obtenidos al compararlos con los propóleos provenientes de otros departamentos de Colombia permite pensar en un escenario diferente para el manejo, la producción y la generación de normativas en nuestro país. Adicionalmente, los extractos etanólicos analizados presentaron alto contenido de fenoles (> 50mg GAE/g EEP), lo cual, se relaciona con la buena actividad antioxidante evaluada, a través de los cuatro métodos para las muestras de Galapa y de Santo Tomás.

Basados en estas observaciones, se debe generar una discusión en torno a los parámetros de calidad que representen apropiadamente los propóleos colombianos, con miras a establecer una normatividad que conduzca a consolidar la industria apícola nacional. Por último y, no por eso menos importante, se hace evidente la necesidad de establecer cuáles son las especies de abejas que producen el propóleos, identificar las plantas empleadas por estas para la elaboración de la resina y determinar las propiedades biológicas de las muestras y los compuestos responsables de la actividad.

Agradecimientos. Los autores agradecen a los laboratorios de Separaciones Químicas y Productos Naturales de la Universidad Nacional, por facilitar el empleo de equipos y la disposición de reactivos. Conflicto de intereses: Los autores declaramos que no existe ningún conflicto de intereses que ponga en riesgo la validez de los resultados presentados.

\section{BIBLIOGRAFÍA}

1. AHN, M.R.; KUMAZAWA, S.; HAMASAKA, T.; BANG, K.; NAKAYAMA, T. 2004. Antioxidant Activity and Constituents of Propolis Collected in Various Areas of Korea. J. Agric. Food Chem. 52(24):7286-7292.

2. AHN, M.R.; KUMAZAWA, S.; USUI, Y.; NAKAMURA, J.; MATSUKA, M.; ZHU, F.; NAKAYAMA, T. 2007. Antioxidant activity and constituents of propolis collected in various areas of China. Food Chem. 101(4):13831392.

3. AUBAD, P.; ROJANO, B.; LOBO, T. 2007. Actividad antioxidante en musgos. Scientia Et Technica. 13(33):23-26.

4. BANKOVA, V. 2005a. Recent trends and important developments in propolis research. eCAM. 2(1):29-32.

5. BANKOVA, V. 2005b. Chemical diversity of propolis and the problem of standardization. J. Ethnopharmacol. 100(1-2):114-117.

6. BANKOVA, V.; CASTRO, S.; MARCUCCI, M. 2000. Propolis: Recent advances in chemistry and plant origin. Apidologie. 31(1):3-15.

7. BANSKOTA, A.; TEZUKA, Y.; ADNYANA, I.; ISHII, E.; MIDORIKAWA, K.; MATSUSHIGE, K.; KADOTA, S. 2001. Hepatoprotective and anti-Helicobacter pylo$r i$ activities of constituents from Brazilian propolis. Phytomedicine. 8(1):16-23.

8. BENZIE, I.; STRAIN J. 1996. The ferric reducing ability of plasma (FRAP) as a measure of "antioxidant power": The FRAP assay. Anal. Biochem. 239(1):7076.

9. BERMÚDEZ-CAMPS, I.; REYES-HERNÁNDEZ, I.; LEÓN-FERNÁNDEZ, O. 2000. Evaluación de la actividad antioxidante del propóleos de la región de Manzanillo. Provincia Granma. Cuba. Bioquimia. 25(3):69-74.

10. CARPES, S.; MOURÃO, G.; DE ALENCAR, S.; MASSON, M. 2009. Chemical composition and free radical scavenging activity of Apis mellifera bee pollen from Southern Brazil. Braz. J. Food Technol. 12(3):220229.

11. CUNHA, I.; SAWAYA; A.; CAETANO, F.; SHIMIZU, M.; MARCUCCI, M.; DREZZA, F.; POVIA, G.; CARVAL- 
HO, P. de O. 2004. Factors that influence the yield and composition of Brazilian propolis extracts. J. Braz. Chem. Soc. 15(6):964-970.

12. FERREIRA-BASTOS, E.; GUZMÁN, D.; FIGUEROA, J.; TELLO, J.; DE OLIVEIRA SCOARIS, D. 2011. Caracterización antimicrobiana y fisicoquímica de propóleos de Apis mellifera l. (hymenoptera: apidae) de la región andina colombiana. Acta biol. Colomb. 16(1):175-184.

13. HUANG, D.; OU, B.; PRIOR, R. 2005. The chemistry behind antioxidant capacity assays. J. Agric. Food Chem. 53(6):1841-1856.

14. KALOGEROPOULOS, N.; KONTELES, S.; TROULLIDOU, E.; MOURTZINOS, I.; KARATHANOS, V. 2009. Chemical composition, antioxidant activity and antimicrobial properties of propolis from Greece and Cyprus. Food Chem. 116(2):452-461.

15. KOO, H.; ROSALEN, P.; CURY, J.; PARK, Y.; IKEGAKI, M.; SATTLER, A. 1999. Effect of Apis mellifera propolis from two Brazilian regions on caries development in desalivated rats. Caries Res. 33(5):393-400.

16. KUJUMGIEV, A.; TSVETKOVA, I.; SERKEDJIEVA, Y.; BANKOVA, V.; CHRISTOV, R.; POPOV, S. 1999. Antibacterial, antifungal and antiviral activity of propolis of different geographic origin. J. Ethnopharmacol. 64(3):235-240.

17. KUMAZAWA, S.; HAMASAKA, T.; NAKAYAMA, T. 2004. Antioxidant activity of propolis of various geographic origins. Food Chem. 84(3):329-339.

18. LIBÉRIO, S.; PEREIRA, A.; ARAÚJO, M.; DUTRA, R.; NASCIMENTO, F.; MONTEIRO-NETO, V.; RIBEIRO, M.; GONÇALVES, A.; GUERRA, R. 2009. The potential use of propolis as a cariostatic agent and its actions on mutans group streptococci. J. Ethnopharmacol. 125(1):1-9.

19. MARCUCCI, M. 1995. Propolis: chemical composition, biological properties and therapeutic activity. Apidologie. 26(2):83-99.

20. MCLENNAN, S.; BONNER, J.; MILNE, S.; LO, L.; CHARLTON, A.; KURUP, S.; JIA, J.; YUE, D.; TWIGG, S. 2008. The anti-inflammatory agent Propolis improves wound healing in a rodent model of experimental diabetes. Wound Repair Regen. 16(5):706-713.
21. MINISTERIO DE AGRICULTURA DE BRASIL-APACAMEAssociação Paulista de Apicultores Criadores de Abelhas Melíferas Européias. 1999. Regulamentos técnicos para fixação de identidade e qualidade de própolis. Revista Mensagem Doce. 52(3):13-14.

22. MIYATAKA, H.; NISHIKI, M.; MATSUMOTO, H.; FUJIMOTO, T.; MATSUKA, M.; SATOH, T. 1997. Evaluation of propolis I. Evaluation of Brazilian and Chinese propolis by enzymatic and physico-chemical methods. Biol. Pharm. Bull. 20(5):496-501.

23. NARANJO, M.; VÉlEZ, T.; ROJANO, B. 2011. Actividad antioxidante de café colombiano de diferentes calidades. Revista Cubana de Plantas Medicinales. 16(2):164-173.

24. NORMA IRAM-INTA 15935-1 Scheme 1. 2004. Instituto Argentino de Normalización-Subcomité de productos agroalimentarios del NOA. Buenos Aires, Argentina.

25. ORTEGA, N.S.; BENITEZ-CAMPO, N.; CABEZAS-FAJARDO, F. 2011. Antibacterial activity and cualitative composition propolis from two climatic regions Cauca department. Biotecn. Sector Agropec. Agroind. 9(1):8-16.

26. OU, B.; HAMPSCH-WOODILL, M.; PRIOR, R. 2001. Development and validation of an improved oxygen radical absorbance capacity assay using fluorescein as the fluorescent probe. J. Agr. Food. Chem. 49(10):4619-4626.

27. PALOMINO-GARCÍA, L.; GARCÍA, C.; GIL, J.; ROJANO, B.; DURANGO, D. 2009. Determinación del contenido de fenoles y evaluación de la actividad antioxidante de propóleos recolectados en el Departamento de Antioquia (Colombia). Vitae. 16(3):388-395.

28. PALOMINO-GARCÍA, L.; MARTÍNEZ-GALÁN, J.; GARCÍA-PAJÓN, C.; GIL-GONZÁLEZ, J.; DURANGORESTREPO, D. 2010. Caracterización fisicoquímica y actividad antimicrobiana del propóleos en el Municipio de la Unión (Antioquia, Colombia). Rev. Fac. Nacional de Agronomía - Medellín. 63(1):53735383.

29. PETROVA, A.; POPOVA, M.; KUZMANOVA, C.; TSVETKOVA, I.; NAYDENSKI, H.; MULI, E.; BANKOVA, V. 2010. New biologically active compounds from Kenyan propolis. Fitoterapia. 81(6):509-514.

30. POPOVA, M.; TRUSHEVA, B.; ANTONOVA, D.; CUTAJAR, S.; MIFSUD, D.; FARRUGIA, C.; TSVETKOVA, 
I.; NAJDENSKI, H.; BANKOVA, V. 2011. The specific chemical profile of Mediterranean propolis from Malta. Food Chem. 126(3):1431-1435.

31. ROJANO, B.; SAEZ, J.; SCHINELLA, G.; QUIJANO, J.; VÉLEZ, E.; GIL, A.; NOTARIO, R. 2008. Experimental and theoretical determination of the antioxidant properties of isoespintanol (2-isopropyl-3,6-dimethoxy5-methylphenol). J. Mol. Struct. 877(1-3):1-6.

32. RUSSO, V.; CARDILE, V.; SANCHEZ, F.; TRONCOSO, N.; VANELLA, A.; GARBARINO, J.A. 2004. Chilean propolis: antioxidant activity and antiproliferative action in human tumor cell lines. Life Sci. 76(5):545558.

33. SALAMANCA-GROSSO, G.; CORREA-CARVAJAL, I.V.; PRINCIPAL, J. 2007. Perfil de flavonoides e índices de oxidación de algunos propóleos colombianos. Zoot. Trop. 25(2):95-102.

34. SAWAYA, A.; DA SILVA CUNHA, I.; MARCUCCI, M. 2011. Analytical methods applied to diverse types of Brazilian propolis. Chem. Centr. J. 5(1):27.

35. SFORCIN, J. 2007. Propolis and the immune system: a review. J. Ethnopharmacol. 113(1):1-14.

36. SFORCIN, J.; BANKOVA, V. 2011. Propolis: Is there a potential for the development of new drugs?. J. Ethnopharmacol. 133(2):253-260.
37. SILVA, V.; GENTA, G.; MÖLLER, M.; MASNER, M.; THOMSON, L.; ROMERO, N.; RADI, R.; FERNANDES, D.; LAURINDO, F.; HEINZEN, H.; FIERRO, W.; DENICOLA, A. 2011. Antioxidant activity of uruguayan propolis. In vitro and cellular assays. J. Agric. Food Chem. 59(12):6430-6437.

38. SINGLETON, V.; ROSSI Jr, J. 1965. Colorimetry of total phenolics with phosphomolybdic-phosphotungstic acid reagents. Ame. J. Enol. Vitic. 16(3):144-158.

39. VALENTE, M.; BALTAZAR, A.; HENRIQUE, R.; ESTEVINHO, L.; CARVALHO, M. 2011. Biological activities of Portuguese propolis: Protection against free radical-induced erythrocyte damage and inhibition of human renal cancer cell growth in vitro. Food Chem. Toxicol. 49(1):86-92.

40. YANG, H.; DONG, Y.; DU, H.; SHI, H.; PENG, Y.; LI, X. 2011. Antioxidant Compounds from Propolis Collected in Anhui, China. Molecules. 16(4):3444-3455.

Recibido: Noviembre 2 de 2011

Aceptado: Agosto 24 de 2012 